\title{
Study on a Novel Hybrid Intelligent Fault Diagnosis Method Based on Improved DE and RBFNN
}

\author{
Liu Yi \\ School of Mechanical and Electronic Engineering, Wuhan Donghu University, \\ Wuhan, 430212, China
}

\begin{abstract}
The radial basis function neural network (RBFNN) is a great potential artificial intelligence technology and can effectively realize the fault diagnosis for small sample and nonlinear problem. But the parameters of RBFNN model seriously affects the generalization ability and diagnosis accuracy on the great extent. So an improved differential evolution algorithm based on dynamic adaptive adjustment strategy is proposed to optimize the parameters of RBFNN model for obtaining the optimal RBFNN (DASDERBFNN) method. Then the proposed DASDERBFNN method is used to construct a new fault diagnosis (DSDRBFNFD) method. In the DSDRBFNFD method, the dynamic adaptive adjustment strategy is used to adaptively adjust the crossover probability ( $C R$ ) value according to the fitness value of current individual in the population for obtaining the improved DE(DASDE) algorithm. Then the selection of parameters in the RBFNN is regarded as a combination optimization of parameters in order to establish the objective function of combination optimization. The DASDE algorithm is used to search for the optimal value of objective function to obtain the better parameter optimization of the RBFNN (DASDERBFNN), which is applied in the fault diagnosis for constructing a new fault diagnosis (DSDRBFNFD) method. Finally, the proposed DSDRBFNFD method is used to diagnose the fault of the cylinder of the engine in order to validate the diagnosis effectiveness of the DSDRBFNFD method. The experiment results show that the proposed DSDRBFNFD method can obtain the higher accuracy of fault diagnosis and is effective fault diagnosis for the engine.
\end{abstract}

Keywords: engine; differential evolution; dynamic adaptive adjustment strategy; RBF neural network; intelligent fault diagnosis

\section{Introduction}

With the rapid development of large-scale and complex modern industrial equipment, these equipments will lead to increase the possibility of fault and maintenance, which brings great economic losses to enterprises. Therefore, it is very important to study the fault diagnosis technology.

Fault diagnosis technology is a kind of state recognition technology based on the current status of the equipment and the historical status of the equipment. It is a state identification technology by using signal processing analysis method for evaluating the equipment status. The traditional fault diagnosis technology has a better effect on the analysis of the deep level fault of the complex structure, and the higher ability for the operator. In recent years, with the rapid development of artificial intelligence technology, the fault diagnosis technology is developed from the traditional technology to the intelligent technology [1,2]. The intelligent fault diagnosis technology is a kind of intelligent diagnosis methods based on knowledge processing technology. It can realize the integration of dialectical logic and mathematical logic, the unification of symbolic processing and numerical processing and reasoning process and algorithm process by the concept and process knowledge in order to realize the intelligent fault diagnosis method 
of equipment. The intelligent fault diagnosis method provides people with the powerful tool for solving the fault diagnosis problem of complex system [3-5].

At present, a lot of scholars have studied the field of intelligent fault diagnosis and proposed a variety of intelligent fault diagnosis technologies. Momoh et al. [6] proposed an integrated package for fault diagnosis in either grounded or ungrounded distribution systems. It utilizes rule based schemes as well as artificial neural networks (ANN) to detect, classify and locate faults. Yang et al. [7] proposed an intelligent fault diagnosis based on fuzzy neural network (NN) expert system for a type of missile weapon system, the concrete implementation of a fuzzy NN fault diagnosis expert system is given. Yang et al. [8] proposed a new approach for integrating case-based reasoning (CBR) with an ART-Kohonen neural network (ART-KNN) to enhance fault diagnosis. Lee et al. [9] proposed an effective fault location algorithm and intelligent fault diagnosis scheme. Chen and Mo [10] proposed an intelligent methodology for diagnosing incipient faults in rotating machinery. In this fault diagnosis system, wavelet transform techniques are used in combination with a function approximation model to extract fault features. Bai et al. [11] focused on the development of an intelligent diagnostic system for rotating machinery. The system is composed of a signal processing module and a state inference module. Widodo et al. [12] proposed the application of independent component analysis (ICA) and support vector machines (SVMs) to detect and diagnose of induction motor faults. . Various scenarios are examined using data sets of vibration and stator current signals from experiments, and the results are compared to get the best performance of classification process. Chai et al. [13] proposed an innovative work-situation fault diagnosis (WSFD) and fault-tolerance control (FTC) strategy for a control system where a combination of neural networks, expert system. Lei et al. [14] proposed a new approach to intelligent fault diagnosis based on statistics analysis, an improved distance evaluation technique and adaptive neuro-fuzzy inference system (ANFIS). The proposed approach is applied to fault diagnosis of rolling element bearings, and testing results show that the proposed approach can reliably recognise different fault categories and severities. Widodo et al. [15] proposed a method for induction motor fault diagnosis based on transient signal using component analysis and support vector machine (SVM). Xian and Zeng [16] proposed a new intelligent method for the fault diagnosis of the rotating machinery based on wavelet packet analysis (WPA) and hybrid support machine (hybrid SVM). Mollazade et al. [17] proposed a fault diagnosis method based on a fuzzy inference system (FIS) in combination with decision trees. Experiments were conducted on an external gear hydraulic pump. Deng et al. [18] proposed a novel complex intelligence fault diagnosis model based on rough sets (RS), artificial neural networks (ANN) with expert system (ES) in distribution substation. Huang et al. [19] proposed a new method based on improved empirical mode decomposition (EMD) energy entropy and multi-class support vector machine (MSVM) to diagnose fault for high voltage CB. Wang and Chen [20] proposed an intelligent diagnosis method for a rolling element bearing; the method is constructed on the basis of possibility theory and a fuzzy neural network with frequency-domain features of vibration signals. Shen et al. [21] proposed a novel model for fault diagnosis based on empirical mode decomposition (EMD) and multi-class transductive support vector machine (TSVM), which is applied to diagnose the faults of the gear reducer. Guo et al. [22] proposed a novel analog circuit fault diagnosis approach based on greedy kernel principal component analysis (KPCA) and one-against-rest support vector machine (OARSVM). Luo et al. [23] proposed an agent oriented intelligent fault diagnosis system. Li et al. [24] proposed an intelligent diagnostic technique based on the integration of the empirical mode decomposition (EMD), kernel independent component analysis (KICA), Wigner bispectrum and support vector machine (SVM). Dou et al. [25] proposed a new method for intelligent fault identification of rotating machinery based on the empirical mode decomposition (EMD), dimensionless parameters, fault decision table (FDT), MLEM2 rule induction algorithm and improved rule matching strategy (IRMS). Zhang et 
al. [26] proposed a new approach for rotating machinery which integrates wavelet transform (WT), principal component analysis (PCA), and artificial neural networks (ANN) to classify the fault and predict the conditions of components, equipment, and machines. Xiang and Cui [27] proposed a remote fault diagnosis system based on combining of expert system and artificial neural networks for monitoring fault occurrence and diagnosing fault. Deng et al. [28] proposed a novel hybrid intelligence method based on integrating rough set, genetic algorithms, and radial basic function neural network (RGRN) in order to improve the correctness and efficiency of fault diagnosis. Krishnakumari and Elayaperumal [29] proposed two different online condition monitoring systems using fuzzy and artificial neural network (ANN) controller for the fault diagnosis of spur gear. Wang et al. [30] proposed a noise-based intelligent method based on the techniques of Hilbert-Huang transform (HHT) and support vector machine (SVM) for engine fault diagnosis (EFD), so-called HHT-SVM mode. Shang, et al. [31] proposed a new automatic fault detection method for automobile transmission and a fault diagnosis expert system for newly assembled transmission, related method of knowledge representation, feature extraction and fault classification is given. Zhang, et al. [32] proposed a novel fault diagnosis strategy based on rotor dynamics and computational intelligence in order to achieve accurate diagnosis for rotating machinery automatically and obtain test data under actual fault conditions. Zhang et al. [33] proposed a novel intelligent fault diagnosis method with multivariable ensemble-based incremental support vector machine (MEISVM), which is testified on a benchmark of roller bearing experiment in comparison with other methods. Aydn et al. [34] proposed a new combined methods based on multiple wireless sensor system for real-time condition monitoring of electric machines. Zou and Huang [35] proposed a new intelligent diagnosis method for classification different conditions of electrical equipment using data obtained from infrared images. Qu et al. [36] proposed a novel intelligent method based on dual-tree complex wavelet packet transform (DTCWPT) and multiple classifier fusion. Gopinath et al. [37] proposed an intelligent fault diagnosis of synchronous generators.

Although these proposed fault diagnosis methods can better realize the fault diagnosis, but the accuracy of fault diagnosis is more lower. The RBFNN model can effectively realize the fault diagnosis for small sample and nonlinear problem. But the parameters of RBFNN model seriously affects the generalization ability and diagnosis accuracy. So an improved differential evolution algorithm based on dynamic adaptive adjustment strategy is proposed to optimize the parameters of RBFNN model for obtaining the optimal RBFNN(DASDERBFNN) method. Then the proposed DASDERBFNN method is used to construct a new fault diagnosis (DSDRBFNFD) method. Finally, the proposed DSDRBFNFD method is used to diagnose the fault of the cylinder of the engine in order to validate the diagnosis effectiveness of the DSDRBFNFD method.

\section{RBF Neural Network}

Artificial neural network (ANN) [38] is a great potential artificial intelligence technology, which is composed of a large number of nerve cells. The ANN provides the significant support for organizing, classifying, and summarizing data. It also helps to discern patterns among input data, require few assumptions, and achieve a high degree of accuracy for fault diagnosis. These advantages of ANN make the neural network technology with a potentially promising alternative tool for realizing the recognition, classification, and fault diagnosis with the accuracy, adaptability, robustness, effectiveness, and efficiency. The structure of ANN usually includes the input layer, hidden layer and output layer, shown in Figure 1. 


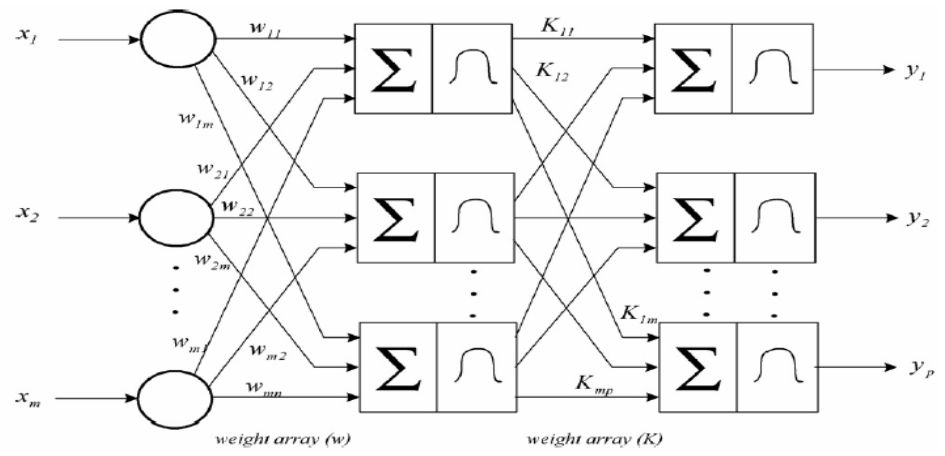

Figure 1. The Structure of ANN

In the ANN, it contains very simple and highly interconnected processors, called neurons. The neurons are used to connect each other by the weighted links in order to transfer the signal from one layer to the other layer. The input layer is represented by circles and behaves as a buffer. Each neuron receives multiple inputs from other neurons, except for the neurons in the input layer, in proportion to their connection weights and then generates a single output in accordance with an activation function. An activation function can be linear or nonlinear form depending on applications. The ANN is trained in order to adjust the network weights by using the different learning algorithm. The training speed and real-time of the neural network are considered in order to be more adaptable for fault diagnosis. Because radial basis function neural network (RBFNN) takes on quick learning speed, so the RBFNN is selected to implement fault diagnosis in this paper. The RBFNN is to belong to the multi-layer forward neural network, shown in Figure 2.

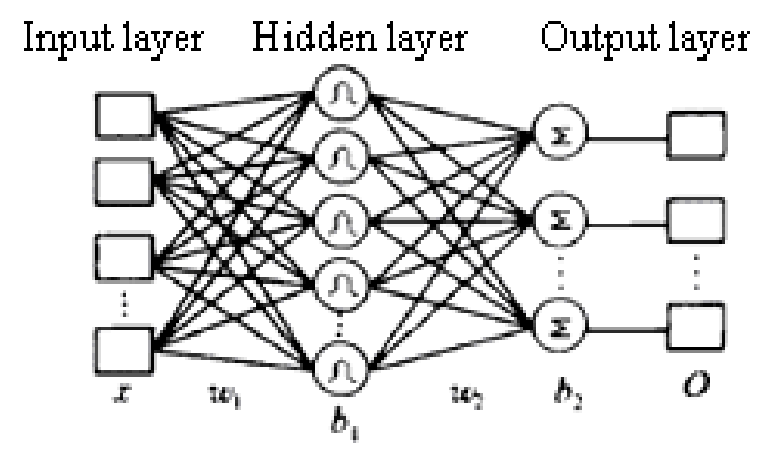

Figure 2. The Structure of RBF Neural Network

As can be known from Figure 2, when the input learning vector of the RBFNN is $x=\left\{x_{1}, x_{2}, \ldots, x_{n}\right\} T \in R^{n}$, the output of the neuron $k$ in the output layer can be expressed as

$$
f(x)=\sum_{i=1}^{m} w_{i} \phi\left(x, c_{i}\right)
$$

where $f(x)$ is output vector, $x$ is a N-dimension input vector, $\phi\left(x, c_{i}\right)$ is a basis function and the output of the neuron ${ }^{i}$ in the hidden layer. ${ }^{\phi}$ is radial basis function, ${ }^{c}{ }_{i}$ is the centre of the $i^{\text {th }}$ basis function with the same dimension as $x ; w=\left\{w_{1}, w_{2}, \ldots, w_{m}\right\}$, $T \in R^{m}$ is output weight matrix, $m$ is the number of the neuron in the input layer, $n$ is 
the number of the neuron in the hidden layer and $k$ is the number of the neuron in output layer. Gauss function is used to act the basis function, then

$$
\phi\left(x, c_{i}\right)=\exp \left(\frac{-\left\|\mathrm{x}-\mathrm{c}_{\mathrm{i}}\right\|^{2}}{2 \sigma_{i}{ }^{2}}\right)
$$

where ${ }^{\sigma_{i}}$ is the variance of Gauss samples. In the general, the learning algorithm of RBFNN uses a novel dynamic nearest neighbor-Clustering algorithm. The algorithm can generate the least nodes and high learning speed. So the key of the RBFNN is to determine the number of hidden nodes and width of corresponding center nodes.

\section{Differential Evolution Algorithm}

Differential evolution(DE) algorithm[39] is a computational intelligent method by simulating the biological evolution of natural population. The main idea is: the cooperation, competition and the generational evolution and reproduction of individuals in a population are used to improve the adaptation degree of individuals to the external environment, so as to approximate the optimal solution of the problem. In essence, the DE algorithm is a greedy genetic algorithm with high quality thought based on real coding. The flow of the DE algorithm is shown in Figure 3.

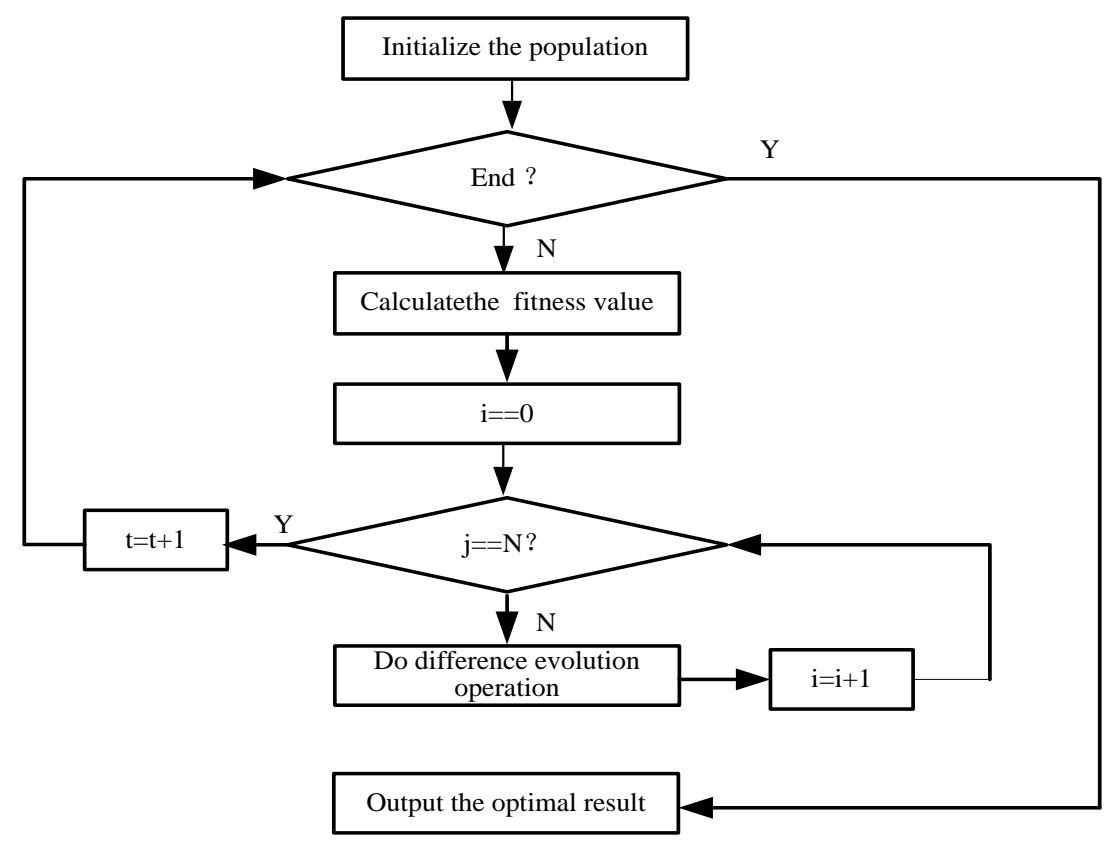

Figure 3. The Flow of DE Algorithm

\section{(1) Initialization}

The parameters of the DE algorithm are initialized in here. These parameters include the population size, the mutation factor, the crossover rate and the stopping criterion. The initial generation counter is set $t=1$. Each individual is encoded as one vector of floatingpoint number. The upper bound and lower bound of each decision variable are initialized according to the uniform probability distribution in the $\mathrm{N}$-dimensional space. The following equation is used to initialize the initial population:

$$
x_{j}(0)=x_{j}^{L}+\operatorname{rand}(0,1) \times\left(x_{j}^{U}-x_{j}^{L}\right){ }_{(j=0,1,2, \cdots, D)}
$$




\section{(2) Mutation Operator}

The DE algorithm executes the mutation to target vector by using differential strategy. The mutation component is the different vector of the parent. Each vector consists of two different individuals $\left(x_{1}, x_{2}\right)$. According to the different generation method of mutation individual, there proposed some different DE algorithms.

(1) $\mathrm{DE} / \mathrm{rand} / 1$

$$
x_{k}=x_{3}+F \times\left(x_{1}-x_{2}\right)
$$

(2) $\mathrm{DE} / \mathrm{rand} / 2$

$$
x_{k}=x_{3}+F \times\left[\left(x_{1}-x_{2}\right)+\left(x_{4}-x_{5}\right)\right]
$$

(3) $\mathrm{DE} / \mathrm{best} / 1 / \mathrm{bin}$

$$
x_{k}=x_{b e s t}+F \times\left(x_{1}-x_{2}\right)
$$

(4) $\mathrm{DE} / \mathrm{rand} / 2$

$$
x_{k}=x_{\text {best }}+F \times\left[\left(x_{1}-x_{2}\right)+\left(x_{4}-x_{5}\right)\right]
$$

(5) $\mathrm{DE} /$ current-to-best/1

$$
x_{k}=x_{3}+F \times\left[\left(x_{1}-x_{2}\right)+\left(x_{\text {best }}-x_{3}\right)\right]
$$

\section{(3) Crossover Operator}

The crossover operation is used to obtain an offspring by mixing current components. In order to enhance the potential diversity of the population, the crossover operation plays a important role. The crossover operation is to execute the uniform crossover between the generated individual $\left({ }^{x_{k}}\right)$ in the mutation and the $i^{\text {th }}$ individual $\left({ }^{x_{i}}\right)$ in the population for compensating the mutation search in the previous step to generate test vector $\left({ }^{x_{G}}\right)$. It includes the binomial cross method and index cross method. The binomial cross method is used in this paper. The crossover operator equation is described as follow:

$$
x_{G j}=\left\{\begin{array}{ll}
x_{k j} & \operatorname{rand}(0,1) \leq C R \text { or } j=j_{\text {rand }} \\
x_{i j}^{t} & \text { other }
\end{array} j=1,2, \cdots, D\right.
$$

where $j_{\text {rand }} \in\{1,2, \cdots, D\}$ is a random integer in order to ensure that at least one component in target individuals $x_{i}$ makes the crossover operation. $x_{i j}$ is the $i^{\text {th }}$ individual of $j^{\text {th }}$ real-valued vector, $x_{k j}$ is the $i^{\text {th }}$ individual of $j^{\text {th }}$ real-valued vector of a mutant vector, $\operatorname{rand}(0,1)$ is the $j^{\text {th }}$ evaluation of the uniform random number, the value of $C R$ is $[0,1]$.

\section{(4) Selection Operator}

The selection operator is used to obtain one population by selecting the trial vectors and their predecessors according to the better fitness value. The DE algorithm can generate the offspring by the greedy selection strategy. After the crossover operation is done, the test individual will be competed between $x_{G}$ and $x_{i}$. The individual of the better fitness value will be selected as the offspring. If the objective function is to be minimized, the selected operation equation is described as follow:

$$
x_{i+1}= \begin{cases}x_{G} & f\left(x_{T}\right)<f\left(x_{i}\right) \\ x_{i} & f\left(x_{T}\right) \geq f\left(x_{i}\right)\end{cases}
$$




\section{An Improved DE (DASDE) Based on Dynamic Adaptive Adjustment Strategy}

Adaptive crossover probability strategy is used to adaptively adjust the crossover probability ( $C R$ ) value according to the fitness value of current individual in the population. If the value of $C R$ is more larger, the more components will be obtained from the mutation vector ${ }^{x_{k}}$. And the ${ }^{x_{k}}$ can realize the more contributions to the vector ${ }^{x_{G}}$. If the value of $C R$ is more lesser, the more components will be obtained from the mutation vector $^{x_{i}}$. And the $x_{i}$ makes much contribution to the vector $X_{G}$. When the $C R$ value is equal to one, the new individual completely comes from the mutation vector ${ }^{x_{k}}$. If the value of $C R$ is zero, the new individual completely comes from the mutation vector ${ }^{x_{i}}$. It will be beneficial to the global search and keeping the population diversity. If the value of $C R$ is one, the new individual completely comes from the mutation vector ${ }^{x_{k}}$. It will be beneficial to the local search and accelerating the convergence. So the value of $C R$ should be smaller in the initial stage in order to improve the global search ability. The value of $C R$ should be gradually increase in the later stage in order to improve the local search ability. So a dynamic adaptive adjustment strategy is used to control the value of $C R$ in this paper. The dynamic adaptive adjustment strategy is described as follow:

$$
C R(t)=\frac{C R_{\min }}{1+\left(\frac{C R_{\min }}{C R_{\max }}-1\right) e^{-\beta t}}
$$

where the $C R_{\min }$ and $C R_{\max }$ are the minimum value and maximum value of crossover probability. The $\beta$ is the initial growth rate, $\beta \in[0,+\infty)$. And $t$ is number of iteration. With the increasing number of iteration, the $\beta$ value is dynamically adjusted. In order to keep the balance between the diversity and convergence, the value of $C R$ is [0.5,1].

\section{The Optimization of RBFNN Based on DASDE Algorithm}

In the learning process of RBF neural network, three important parameters are determined. They are the center, the width and the weight of the network in the basis function of the hidden unit. At present, the number of hidden units is determined by using the experiences. However, the reasonable and accurate selection parameters can realize the nonlinear approximation ability of RBF neural network. The improved DE(DASDE) algorithm has strong optimization performance, it takes on the good robustness and parallel searching advantage. In order to obtain the better parameter optimization of the RBFNN, the DASDE algorithm is used to optimize the parameters of the RBFNN in order to obtain the optimal RBFNN(DASDERBFNN) method. The selection of parameters in the RBFNN is regarded as a combination optimization of parameters in order to establish the objective function of combination optimization. The DASDE algorithm is used to search for the optimal value of objective function. The optimization flow of RBFNN based on DASDE algorithm is described in Figure 4. 


\section{Fault Diagnosis and Case Analysis}

\subsection{Fault Diagnosis Method}

In the past decades, various fault diagnosis methods have been proposed for realizing the fault diagnosis. These methods include the logical reasoning, artificial neural networks, rough set theory, fuzzy logic, expert system, genetic algorithm, particle swarm optimization, ant colony optimization algorithm and other new algorithms. However each method possesses stronger diagnosis ability for small-scale system. For the large-scale system, each method still can not effectively implement diagnosis. So the improved DE(DASDE) algorithm used to optimize the parameters of the RBFNN in order to obtain the better parameter optimization of the RBFNN, then the optimal RBFNN(DASDERBFNN) method is formed in this paper. And the proposed DASDERBFNN method is used to construct a new fault diagnosis (DSDRBFNFD) method in this paper.

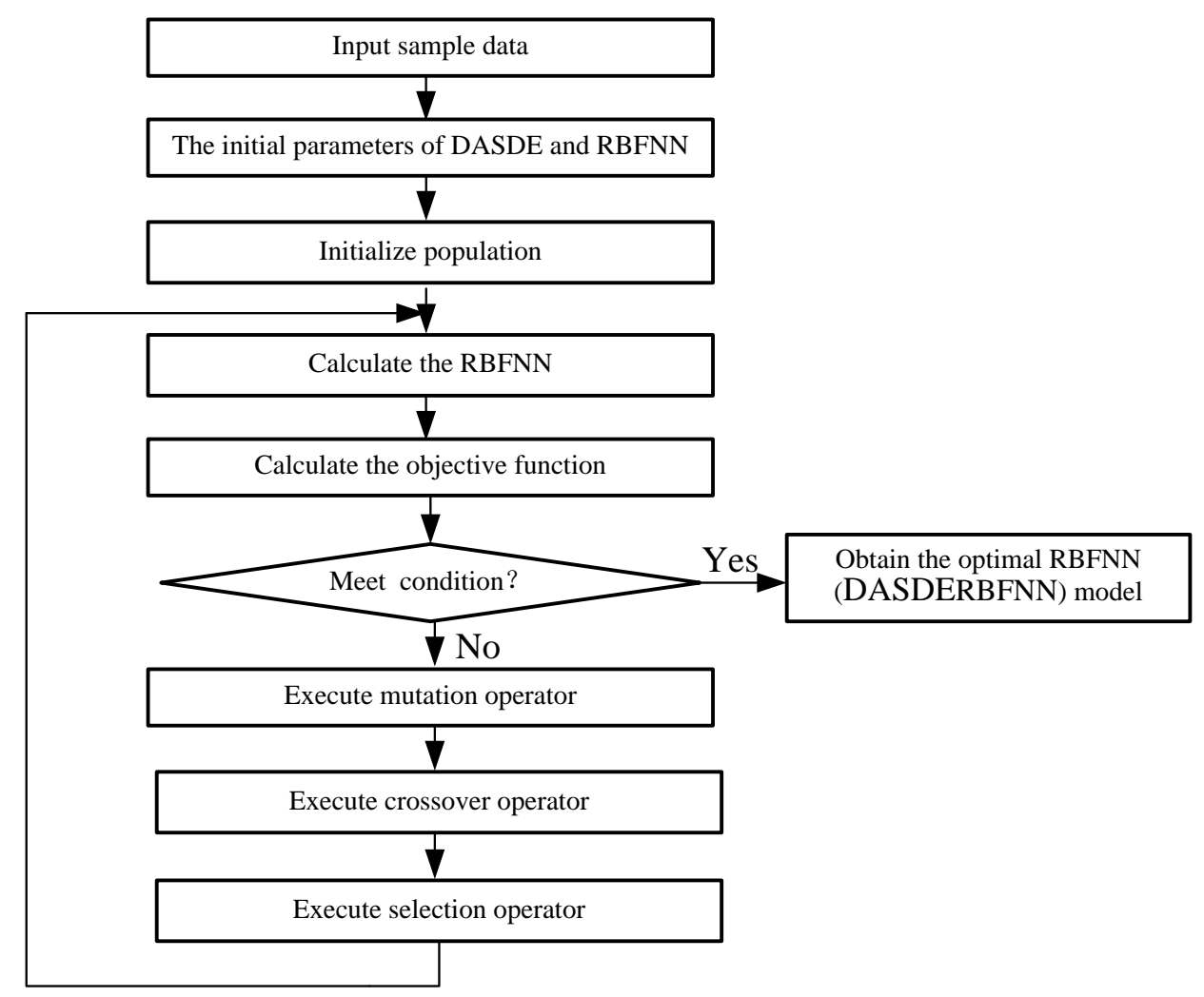

Figure 4. The Flow of DASDERBFNN Algorithm

\subsection{A Case Analysis of the DSDRBFNFD Method}

Engine is a kind of machine, which can transform other types of energy into mechanical energy. It includes gasoline engine, Sterling engine, steam engine, motor and so on. The engine is suitable for power generation device, and can also refer to the whole machine with power plant. In the operation process of the engine, the oil fault and gas fault are the most fault. Due to the complex structure of the engine, it is difficult to distinguish the causes of the fault. So the proposed DSDRBFNFD method is used to achieve the fault diagnosis of the engine. 
In the operation process of the engine, six characteristic parameters are often selected. These characteristic parameters are the maximum acceleration index(AR), the average acceleration index(MC), the maximum deceleration index(DR), the average deceleration index(MI), the torque harmonic component ratio(TA) and the rising speed of the gas explosion(PI). When the fault diagnosis is diagnosed, the characteristic parameters are extracted at the first. The cylinder of the engine has three faults and one normal state. Three faults are the less oil, valve leakage and cylinder leakage. The four sample data are used to test the DSDRBFNFD method, shown in Table 1. These sample data are not very different. The DSDRBFNFD method is initialized as follows: the input unit number is 6 , the output variable is 4, population size is $N P=100$, scaling factor is $F=0.7$, the number of iteration is $T=500$. The initial crossover probability is $C R_{\min }=0.50$ and $C R_{\max }=1.0$. The DSDRBFNFD method is independently run 30 times. The initial decay rate $\alpha$ and the initial growth rate $\beta$ are 80 . The environment is: Matlab 2012a, running on the Pentium IV, 2.0GB RAM.

Table 1. The Sample Data for Testing

\begin{tabular}{cccccccc}
\hline Index & Cylinder state & AR & MC & DR & MI) & TA & PI \\
\hline 1 & Less oil & 0.112 & 0.104 & 0.034 & 0.089 & 0.083 & 0.098 \\
2 & Valve leakage & 0.114 & 0.131 & 0.019 & 0.084 & 0.122 & 0.199 \\
3 & Cylinder leakage & 0.225 & 0.193 & 0.145 & 0.104 & 0.121 & 0.111 \\
4 & Normal state & 1.002 & 1.003 & 0.998 & 1.002 & 1.005 & 0.998 \\
\hline
\end{tabular}

The test sample data in Table 1 are executed in order to obtain the fault diagnosis results by using the proposed DSDRBFNFD method for one time, shown in Table 2.

Table 2. The Diagnosis Result of the Proposed DSDRBFNFD Method

\begin{tabular}{|c|c|c|c|c|c|c|}
\hline \multirow{2}{*}{$\begin{array}{c}\text { Index } \\
1\end{array}$} & \multirow{2}{*}{$\begin{array}{c}\text { Cylinder state } \\
\text { Less oil }\end{array}$} & \multicolumn{4}{|c|}{ Output the result } & \multirow{2}{*}{$\begin{array}{c}\text { Diagnosis result } \\
\text { Less oil }\end{array}$} \\
\hline & & 0.006 & 0.009 & 0.924 & 0.005 & \\
\hline 2 & Valve leakage & 0.007 & 0.031 & 0.007 & 0.915 & Valve leakage \\
\hline 3 & Cylinder leakage & 0.001 & 0.903 & 0.004 & 0.006 & Cylinder leakage \\
\hline 4 & Normal state & 1.001 & 0.073 & 0.0215 & 0.008 & Normal \\
\hline
\end{tabular}

The DSDRBFNFD method, DERBFNFD method and RBFNFD method are independently run 30 times. The average accuracy of fault diagnosis is show in Table 3. 
Table 3. The Average Accuracy of the Proposed DSDRBFNFD Method

\begin{tabular}{ccccc}
\hline \multirow{2}{*}{ Index } & Cylinder state & \multicolumn{3}{c}{ Average accuracy } \\
\cline { 3 - 5 } & & RBFNFD & DERBFNFD & DSDRBFNFD \\
\hline 1 & Less oil & $80.0 \%$ & $86.7 \%$ & $93.3 \%$ \\
3 & Valve leakage & $76.7 \%$ & $86.7 \%$ & $90.0 \%$ \\
4 & Cylinder leakage & $83.3 \%$ & $90.0 \%$ & $96.7 \%$ \\
\hline
\end{tabular}

From the diagnosis results of Table 2 and Table 3, it can be seen that the proposed DSDRBFNFD method can obtain average accuracy of fault diagnosis $93.3 \%$ for less oil, $90.0 \%$ for valve leakage, $96.7 \%$ for cylinder leakage and $100 \%$ for normal state. The RBFNN based on improved DE algorithm is more close to the output vector. The diagnosis accuracy is significantly higher than that of the RBF neural network and DERBFNFD method. The result shows that the optimized RBF neural network based on improved DE algorithm can improve the accuracy of diagnosis. So the proposed DSDRBFNFD method can obtain the highest average accuracy of fault diagnosis than the RBFNFD method and DERBFNFD method. The results show that the proposed DSDRBFNFD method is effective fault diagnosis for engine.

\section{Conclusion}

The RBFNN model is a great potential artificial intelligence technology with a large number of nerve cells. It can organize, classify, and summarize the data. And It can effectively realize the fault diagnosis for small sample and nonlinear problem. But the parameters of RBFNN model seriously affects the generalization ability and prediction accuracy on the great extent. So an improved differential evolution algorithm based on dynamic adaptive adjustment strategy is proposed to optimize the parameters of RBFNN model for obtaining the optimal RBFNN(DASDERBFNN) method. In the DASDERBFNN method, the dynamic adaptive adjustment strategy is used to adaptively adjust the crossover probability value according to the fitness value of current individual in the population for obtaining the improved DE(DASDE) algorithm, which is used to optimize the parameters in the RBFNN for obtaining the better parameter optimization of the RBFNN. Then a new fault diagnosis (DSDRBFNFD) method is proposed. The cylinder of the engine is used to validate the diagnosis effectiveness of the DSDRBFNFD method. The diagnosis accuracy is significantly higher than that of the RBF neural network and DERBFNFD method. The result shows that the optimized RBF neural network based on improved DE algorithm can improve the accuracy of diagnosis.

\section{References}

[1] W. Deng, R. Chen, B. He, Y. Q. Liu, L. F. Yin and J. H. Guo, "A novel two-stage hybrid swarm intelligence optimization algorithm and application", Soft Computing, vol. 16, no. 10, (2012), pp. 17071722 .

[2] Y. H. Lin, P. Lee and T. P. Chang, "Practical expert diagnosis model based on the grey relational analysis technique", Expert Systems with Applications, vol. 36, no. 2, (2009), pp. 1523-1528.

[3] W. Deng, R. Chen, J. Gao, Y. J. Song and J. J. Xu, "A novel parallel hybrid intelligence optimization algorithm for a function approximation problem", Computers and Mathematics with Applications, vol. 63, no. 1, (2012), pp. 325-336. 
[4] T. T. Le, J. Watton and D. T. Pham, "An artificial neural network based approach to fault diagnosis and classification of fluid power systems", Proceedings of the Institution of Mechanical Engineers. Part I, Journal of systems and control engineering, vol. 211, no. 4, (1997), pp. 307-317.

[5] W. Deng, H. M. Zhao, J. J. Liu, X. L. Yan, Y. Y. Li, L. F. Yin and C. H. Ding, "An improved CACO algorithm based on adaptive method and multi-variant strategies", Soft Computing, vol. 19, no. 3, (2015), pp. 701-713.

[6] J. A. Momoh, L. G. Dias and D. N. Laird, "Implementation of a hybrid intelligent tool for distribution system fault diagnosis", IEEE Transactions on Power Delivery, vol. 12, no. 2, (1997), pp. 1035-1040.

[7] J. Yang, Z. Feng, Z. Zhang and P. Liu, "Study on missile intelligent fault diagnosis system based on fuzzy NN expert system”, Journal of Systems Engineering and Electronics, vol. 12, no. 1, (2001), pp. 82-87.

[8] B. S. Yang, T. Han and Y. S. Kim, "Integration of ART-Kohonen neural network and case-based reasoning for intelligent fault diagnosis", Expert Systems with Applications, vol. 26, no. 3, (2004), pp. 387-395.

[9] S. J. Lee, M. S. Choi, S. H. Kang, B. G. Jin, D. S. Lee, B. S. Ahn, N. S. Yoon, H. Y. Kim and S. B. Wee, "An intelligent and efficient fault location and diagnosis scheme for radial distribution systems", IEEE Transactions on Power Delivery, vol. 19, no. 2, (2004), pp. 524-532.

[10] C. Z. Chen and C. T. Mo, "A method for intelligent fault diagnosis of rotating machinery", Digital Signal Processing: A Review Journal, vol. 14, no. 3, (2004), pp. 203-217.

[11] M. S. Bai, J. M. Huang, M. H. Hong and F. C. Su, "Fault diagnosis of rotating machinery using an intelligent order tracking system", Journal of Sound and Vibration, vol. 280, no. 3-5, (2005), pp. 699718.

[12] A. Widodo, B. S. Yang and T. Han, "Fault diagnosis of rotating machinery using an intelligent order tracking system", Expert Systems with Applications, vol. 32, no. 2, (2007), pp. 299-312.

[13] T. Y. Chai, F. H. Wu, J. L. Ding and C. Y. Su, "Intelligent work-situation fault diagnosis and faulttolerant system for the shaft-furnace roasting process", Proceedings of the Institution of Mechanical Engineers. Part I: Journal of Systems and Control Engineering, vol. 221, no. 6, (2007), pp. 843-855.

[14] Y. G. Lei, Z. J. He, Y. Y. Zi, "A new approach to intelligent fault diagnosis of rotating machinery", Expert Systems with Applications, vol. 35, no. 4, (2008), pp. 1593-1600.

[15] A. Widodo, B. S. Yang, D. D. Gu and B. K. Choi, "Intelligent fault diagnosis system of induction motor based on transient current signal”, Mechatronics, vol. 19, no. 5, (2009), pp. 680-689.

[16] G. M. Xian and B. Q. Zeng, "An intelligent fault diagnosis method based on wavelet packer analysis and hybrid support vector machines", Expert Systems with Applications, vol. 36, no. 10, (2009), pp. 1213112136.

[17] K. Mollazade, H. Ahmadi, M. Omid and R. Alimardani, "An intelligent model based on data mining and fuzzy logic for fault diagnosis of external gear hydraulic pumps", Insight: Non-Destructive Testing and Condition Monitoring, vol. 51, no. 11, (2009), pp. 594-600.

[18] W. Deng, R Chen, X. H. Yang, Y. J. Song and W. Li, “An intelligent fault diagnosis method based on soft computing and expert system", Engineering Intelligent System, vol. 18, no. 2, (2010), pp. 77-84.

[19] J. Huang, X. G. Hu and X. Geng, "An intelligent fault diagnosis method of high voltage circuit breaker based on improved EMD energy entropy and multi-class support vector machine", Electric Power Systems Research, vol. 81, no. 2, (2011), pp. 400-407.

[20] H. Q. Wang and P. Chen, "A Intelligent diagnosis method for rolling element bearing faults using possibility theory and neural network", Computers and Industrial Engineering, vol. 60, no. 4, (2011), pp. 511-518.

[21] Z. J. Shen, X. F. Chen, X. L. Zhang and Z. J. He, “A novel intelligent gear fault diagnosis model based on EMD and multi-class TSVM", Measurement: Journal of the International Measurement Confederation, vol. 45, no. 1, (2012), pp. 30-40.

[22] K. Guo, Y. Zhu and Y. San, "Analog circuit intelligent fault diagnosis based on greedy KPCA and oneagainst-rest SVM approach", Journal of Theoretical and Applied Information Technology, vol. 46, no. 1, (2012), pp. 147-157.

[23] H. Luo, S. L. Yang, X. L. Hu and X. X. Hu, "Agent oriented intelligent fault diagnosis system using evidence theory", Expert Systems with Applications, vol. 39, no. 3, (2012), pp. 2524-2531.

[24] Z. X. Li, X. P. Yan, C. L. Yuan and Z. X. Peng, "Intelligent fault diagnosis method for marine diesel engines using instantaneous angular speed", Journal of Mechanical Science and Technology, vol. 26, no. 8, (2012), pp. 2413-2423.

[25] D. Y. Dou, J. G. Yang, J. T. Liu and Y. K. Zhao, "A rule-based intelligent method for fault diagnosis of rotating machinery", Knowledge-Based Systems, vol. 36, no. 12, (2012), pp. 1-8.

[26] Z. Y. Zhang, Y. Wang and K. S. Wang, "Intelligent fault diagnosis and prognosis approach for rotating machinery integrating wavelet transform, principal component analysis, and artificial neural networks", International Journal of Advanced Manufacturing Technology, vol. 68, no. 1-4, (2012), pp. 763-773.

[27] L. Xiang and W. Cui, "Study of intelligence diagnosis system for wind turbine gearbox fault", Telkomnika - Indonesian Journal of Electrical Engineering, vol. 11, no. 8, (2013), pp. 4691-4697.

[28] W. Deng, X. H. Yang, J. J. Liu, H. M. Zhao, Z. G. Li ad X. L. Yan, "A novel fault analysis and diagnosis method based on combining computational intelligence methods", Proceedings of the 
Institution of Mechanical Engineers, Part E: Journal of Process Mechanical Engineering, vol. 227, no. 3, (2013), pp. 198-210.

[29] A. Krishnakumari and A. Elayaperumal, "Gear fault diagnosis using vibration signals based on decision tree assisted intelligent controllers", Journal of Vibroengineering, vol. 15, no. 4, (2013), pp. 1826-1837.

[30] Y. S. Wang, Q. H. Ma, Q. Zhu, X. T. Liu and L. H. Zhao, "An intelligent approach for engine fault diagnosis based on Hilbert-Huang transform and support vector machine", Applied Acoustics, vol. 75, no. 1, (2014), pp. 1-9.

[31] W. L. Shang, X. F. Zhou and J. Yuan, "An intelligent fault diagnosis system for newly assembled transmission”, Expert Systems with Applications, vol. 41, no. 9, (2014), pp. 4060-4072.

[32] J. H. Zhang, W. P. Ma, J. W. Lin, L. Ma and X. J. Jia, "Fault diagnosis approach for rotating machinery based on dynamic model and computational intelligence", Measurement: Journal of the International Measurement Confederation, vol. 59, no. 1, (2015), pp. 73-87.

[33] X. L. Zhang, B. J. Wang and X. F. Chen, "Intelligent fault diagnosis of roller bearings with multivariable ensemble-based incremental support vector machine”, Knowledge-Based Systems, vol. 89, (2015), pp. 56-85

[34] L. Aydn, M. Karaköse and E. Akn, "Combined intelligent methods based on wireless sensor networks for condition monitoring and fault diagnosis", Journal of Intelligent Manufacturing, vol. 26, no. 4, (2015), pp. 717-729.

[35] H. Zou and F. Z. Huang, "A novel intelligent fault diagnosis method for electrical equipment using infrared thermography", Infrared Physics and Technology, vol. 73, (2015), pp. 29-35.

[36] J. X. Qu, Z. S. Zhang and T. Gong, "A novel intelligent method for mechanical fault diagnosis based on dual-tree complex wavelet packet transform and multiple classifier fusion", Neurocomputing, vol. 171, (2016), pp. 837-853.

[37] R. Gopinath, C. S. Kumar, K. I. Ramachandran, V. Upendranath and P. V. R. S. Kiran, "Intelligent fault diagnosis of synchronous generators", Expert Systems with Applications, vol. 45, no. 3, (2016), pp. 142149.

[38] M. S. Bailai, Z. J. Khan, H. M. Suryawanshi and R. L. Sonolikar, "Application of ANN for the detection of winding insulation condition and bearing wear in single phase induction motor", Engineering Intelligent Systems, vol. 1, no. 16, (2008), pp. 79-93.

[39] S. K Price, "Differential evolution-a simple and efficient heuristic for global optimization over continuous spaces", Journal of Global Optimization, vol. 11, no. 4, (1997), pp. 341-359.

\section{Author}

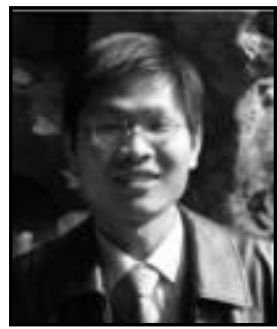

Liu Yi, received the Doctor degree in Industrial engineering from Wuhan University of Technology in 2014, Wuhan, China. The main research directions: Advanced manufacturing system, Multi-agent systems and other Artificial Intelligence. 